\title{
Controlled Quadrature
}

\author{
By Irwin Roman
}

1. Introduction. If $y=f(x)$ is a function for which the integral $F(x)=\int_{a}^{x} f(x) d x$ can be found and evaluated numerically, and if $F(x)$ is continuous on the interval $a \leqq x \leqq b$, then the definite integral of $f(x)$ from $x=a$ to $x=b$ is $I=\int_{a}^{b} f(x) d x$ $=F(b)-F(a)$. Under proper restrictions the property of continuity can be removed, but this extension is not considered in this paper.

If $F(x)$ cannot be found, or if the numerical evaluation is inconvenient, mechanical (numerical) quadrature usually can be used with one or more of the numerous formulas available in the literature. The quadrature value is augmented by a remainder term, usually given as the value of a function at an undetermined point of the interval of integration. Frequently quadrature formulas are applied on the assumption that the remainder term is negligible. This may or may not be verified by establishing an upper bound for the remainder term. If verified, it seldom is used to control the lengths of the subintervals for the integration. Even when subintervals are used, the bounds are determined for the entire interval of integration, not the subintervals.

The choice of a quadrature formula must be made by the user. Each has advantages in some applications. The present discussion uses the Simpson quadrature formula to illustrate the method, which can be applied to other formulas without important changes in the fundamental method, provided the remainder term is obtainable. Simpson's formula has been selected to illustrate the method for several reasons:

1. It involves the values of the integrand at only three points.

2. The only derivative needed is of the fourth order.

3. It allows considerable flexibility in the choice of subintervals, which fact is important if tables of the integrand are limited.

2. Present Method. The method suggested here utilizes both a lower and an upper bound of the remainder term, thus reducing the extent of the uncertainty and determining a mean value for it. The remainder term is determined for each subinterval, not necessarily of a fixed length throughout the entire interval of integration. Before performing the quadrature, the range of the remainder term on each subinterval is determined, by trial and approximation if necessary. The first subinterval has its initial end at $x=a$, and the last subinterval has its terminal end at $x=b$. Each subinterval has its initial end at the terminal end of another interval, so that the coverage of the original interval by the subintervals is complete without overlapping.

Even in those cases for which actual extrema can be found, it often is simpler to

Received June 6, 1963. 
use bounds for the remainder term on an interval. Frequently this will eliminate the need for a precise numerical evaluation of the extrema. The use of bounds merely augments an uncertainty inherent in the remainder term. The term bound is used here in its usual mathematical sense. An upper bound exceeds the value of the function at every point of its interval, and the value of the function exceeds the lower bound at every point. A maximum is a least upper bound of the function and a minimum is a greatest lower bound.

If bounds are selected for the remainder term over a selected interval, the error can be used to determine the lengths of the subintervals, if the possible quadrature error exceeds the accepted tolerance. The total quadrature value and error are the sums of the corresponding values for the subintervals.

If desired, each interval can be determined by successive trial to give an optimum length for which the error does not exceed, but is close to, the accepted tolerance for each interval. However, the extra calculations usually are not justified. Subdividing an interval decreases the total error, as the bounds cannot be increased by subdividing, and at least one of the subintervals can be expected to lead to bounds less separated than in the original interval.

As a routine procedure, the error is evaluated for the entire interval to obtain a basis for subdividing. Although more subintervals may be used, it usually is adequate to divide an interval into two parts after the first subdivision has been made. In examining the errors for the initial subdivision, the number of significant figures may be reduced below that desired in the quadrature value, as the error can be expected to be much smaller than the quadrature value. After the original interval has been subdivided to assure an acceptably small error, the quadrature formula is applied. In selecting the subintervals, the available tables of the integrand or of functions needed in calculating the values of the integrand must be considered, as the values of the integrand or of functions needed in obtaining its values must be available at the ends and center of each subinterval. Otherwise, interpolation or involved computations may be needed. It is preferable to select shorter intervals if that will permit the use of existing tables.

If the range of the remainder term for an interval is acceptably small, its midpoint may be taken as the value of the correction, and the error cannot exceed half of the range of the remainder term. If the range of the remainder term is too great, the interval is subdivided, and the analysis is repeated until the error for each subinterval is acceptably small and the sum of the individual errors is also acceptably small. The method is progressive. At each step those intervals that have errors above the tolerance are subdivided. After each interval error is below the tolerance, further subdivision is needed if the sum of the individual errors exceed the tolerance, in which case the interval that has the largest error term is subdivided. The subdividing continues until the total error is acceptably small.

After the subdivision has been selected by approximate calculation to assure that the total error cannot exceed the tolerance, the quadrature formula is applied. For each subinterval, the correction term, error term, and quadrature value are calculated. The total quadrature value is the sum of the individual quadrature values, and the adjusted value is obtained by adding to the total quadrature value the sum of the corrections, signs considered. The total error cannot exceed the sum 
of the individual errors. It should be remembered that the correction as used here is the arithmetic mean of the signed values of the lower and upper bounds of the remainder term and that the actual error cannot exceed the error as used here.

The method of subdivision and the choice of subintervals are not unique, but the value determined for the integral has an acceptably small error.

As many of the functions involved in the calculation of quadrature errors are in the form of, or contain terms in the form of, products, the bounds of a product are important. The extrema of $u v$ occur for values of the argument for which $u v^{\prime}+u^{\prime} v=0$, which frequently is difficult to solve. However, the bounds of a product must lie on the range determined by the products of the bounds of the factors. If the subscript 1 denotes a lower bound and the subscript 2 denotes an upper bound, it follows that

$$
(u v)_{1} \leqq\left\{u_{1} v_{1}, u_{1} v_{2}, u_{2} v_{1}, u_{2} v_{2}\right\} \leqq(u v)_{2},
$$

where the curly braces indicate that the inequality applies to each element enclosed by them. Specifically, $(u v)_{1}$ may be selected as the algebraically smallest of the four products and $(u v)_{2}$ as the algebraically largest of the four products. Usually this choice can be made by inspection, but occasionally two or more of the products may be so nearly equal that the products must be calculated to determine the acceptable value.

If a function is monotonic on an interval, the bounds may be selected as the values of the function at the ends of the interval. However, if $f(x)$ has a minimum at $x=m$ and/or a maximum at $x=n$, where $a \leqq\{m, n\} \leqq b$, then $f(m) \leqq f(x)$ $\leqq f(n)$ when $a \leqq x \leqq b$ and the lower and upper bounds may be selected as $f_{1} \leqq$ $f(m)$ and $f_{2} \geqq f(n)$, respectively.

This method does not require that all subintervals shall have the same length, nor that they have lengths that follow some preassigned pattern, such as increasing.

In most of the usual quadrature formulas, $I=\int_{a}^{b} f(x) d x=I_{0}+R$, where $I_{0}=\sum_{k=0}^{n} a_{k} f\left(x_{k}\right)$ and $R$ depends on $a, b$, and some function determined from $f(x)$. The values of $a_{k}, x_{k}$, and $n$ depend on the specific quadrature formula used. For Simpson's quadrature [1, Sec. 172], [2, Sec. 57.1] and others, $I_{0}=(\delta / 3)\left(y_{0}+4 y_{1}+y_{2}\right)$, where $\delta=(b-a) / 2$ and $y_{j}=f(a+j \delta)$. The remainder term is $R=-\delta^{5} f^{i v}(\xi) / 90$, where $a \leqq \xi \leqq b$. If $g(x)=f^{i v}(x)$, the lower and upper bounds of $f^{i v}(x)$ are $g_{1}$ and $g_{2}$, respectively, and $R=-\delta^{5} g(\xi) / 90$. The bounds of $R$ are selected algebraically from $-\delta^{5} g_{1} / 90$ and $-\delta^{5} g_{2} / 90$.

If $g_{1}$ is a lower bound and $g_{2}$ an upper bound of $g(x)$ on the interval $a \leqq x \leqq b$, where $b=a+2 \delta$, and if $C=-\delta^{5}\left(g_{1}+g_{2}\right) / 180$ and $E=\delta^{5}\left(g_{2}-g_{1}\right) / 180$, then $I_{c}=I_{0}+C$ will be within $E$ of $I$.

If $f(x)$ is a polynomial of degree not above three, $g(x)=0$, so that $C=E=0$ and $I_{c}=I_{0}=I$, exactly. If $f(x)$ is a polynomial of degree four, $g(x)=k$ is constant so that $C=-\delta^{5} k / 90$ and $E=0$. Accordingly, $I=I_{c}=I_{0}-\delta^{5} k / 90$, exactly. For other functions, the results are approximate.

3. Examples. To illustrate the application of the method, four examples are given, each introducing a feature not included in those that precede it and each omitting details that have been discussed in the previous examples. 
Table 1

\begin{tabular}{|c|c|c|c|}
\hline \multirow{2}{*}{$1-e^{-3}=\underset{n}{0.9502129316}$} & \multicolumn{3}{|c|}{$G=0.005278060731$} \\
\hline & 10 & 12 & 15 \\
\hline $\begin{array}{c}\delta \\
2 \delta \\
\delta^{5} \times 10^{5} \\
e^{-\delta} \\
B=e^{-2 \delta} \\
1-B \\
(1+B) /(1-B) \\
E=G \delta^{5} \\
C=(1+B) E /(1-B) \\
\delta\left(1+4 e^{-\delta}+e^{-2 \delta}\right) / 3 \\
A_{t}\end{array}$ & \begin{tabular}{lll}
\multicolumn{3}{c}{0.15} \\
0.30 \\
7.593 & 750 & 000 \\
0.860 & 707 & 9764 \\
0.740 & 818 & 2207 \\
0.259 & 181 & 7793 \\
6.716 & 591 & 828 \\
0.000 & 000 & 401 \\
0.000 & 002 & 693 \\
0.259 & 182 & 506 \\
3.666 & 202 & 671
\end{tabular} & \begin{tabular}{lll}
\multicolumn{3}{c}{0.125} \\
0.250 \\
3.051 & 757 & 812 \\
0.882 & 496 & 9026 \\
0.778 & 800 & 7831 \\
0.221 & 199 & 2169 \\
8.041 & 623 & 330 \\
0.000 & 000 & 161 \\
0.000 & 001 & 295 \\
0.221 & 199 & 516 \\
4.295 & 733 & 705
\end{tabular} & \begin{tabular}{lll}
\multicolumn{4}{c}{0.1} \\
\multicolumn{4}{c}{0.2} & \\
1.000 & 000 & 000 \\
0.904 & 837 & 4180 \\
0.818 & 730 & 7531 \\
0.181 & 269 & 2469 \\
10.033 & 311 & 13 \\
0.000 & 000 & 053 \\
0.000 & 000 & 532 \\
0.181 & 269 & 348 \\
5.241 & 997 & 459
\end{tabular} \\
\hline $\begin{array}{c}I_{0} \\
I_{c}=I_{0}+C \\
I-I_{c}\end{array}$ & $\begin{array}{lll}0.950 & 215 & 596 \\
0.950 & 212 & 903 \\
0.000 & 000 & 029\end{array}$ & $\begin{array}{lll}0.950 & 214 & 216 \\
0.950 & 212 & 921 \\
0.000 & 000 & 011\end{array}$ & $\begin{array}{lll}0.950 & 213 & 462 \\
0.950 & 212 & 930 \\
0.000 & 000 & 002\end{array}$ \\
\hline
\end{tabular}

Example 1. An example, in which the results can be checked is:

$$
I=\int_{0}^{3} e^{-x} d x=1-0.049787068=0.950212932 .
$$

In this example, $f(x)=e^{-x}$ so that $g(x)=e^{-x}$. For the interval $(a, b), g_{1}=e^{-b}$ and $g_{2}=e^{-a}$, so that $g_{2}-g_{1}=e^{-a}\left(1-e^{-2 \delta}\right)$ and $g_{2}+g_{1}=e^{-a}\left(1+e^{-2 \delta}\right)$. Accordingly, $E=L A$ and $C=-M A$, where $A=e^{-a}, B=e^{-2 \delta}, L=\delta^{5}(1-B) / 180$, and $M=\delta^{5}(1+B) / 180$. The quantities $B, L$, and $M$ depend only on $\delta$, whereas $A$ depends only on $a$. For a selected value of $\delta, E$ and $C$ decrease as $a$ increases. If the total interval $(0,3)$ is divided into $n$ subintervals of the same length, then $\delta=$ $3 /(2 n)=1.5 / n$. For the $k$ th subinterval, $a=2(k-1) \delta$ and $A_{k}=e^{-2(k-1) \delta}$ where $1 \leqq k \leqq n$. For the $k$ th subinterval, the error term is $E_{k}=L A_{k}$ and the correction term is $C_{k}=-M A_{k}$. Accordingly, the error term is $E=L \sum_{k=1}^{n} A_{k}$ and the correction term is $C=-M \sum_{k=1}^{n} A_{k}$, for the entire interval of integration. But

$$
A_{t}=\sum_{k=1}^{n} A_{k}=\frac{1-e^{-3}}{1-e^{-2 \delta}}=\frac{0.9502129316}{1-B} \text {. }
$$

Accordingly, the total error is $E=L A_{t}=0.005278960731 \delta^{5}$, and the total correction is $C=-M A_{t}=-(1+B) E /(1-B)$. The Simpson quadrature value for the $k$ th interval is $I_{k}=(\delta / 3)\left[e^{-a}+4 e^{-(a+\delta)}+e^{-(a+2 \delta)}\right]=(\delta / 3) A_{k}(1+$ $\left.4 e^{-\delta}+e^{-2 \delta}\right)$, so that the total quadrature value is:

$$
I_{0}=(\delta / 3)\left(1+4 e^{-\delta}+e^{-2 \delta}\right) A_{t} .
$$

If the total error is to be less than $5 \times 10^{-7}=L A_{t}=5.278960731 \delta^{5} \times 10^{-3}$, then $\delta^{5} \leqq 9.471561268 \times 10^{-5}$ and $\delta \leqq 0.156$. For $\delta \leqq 0.156 n \geqq 10$. To avoid 


\section{Table 2}

\begin{tabular}{|c|c|c|c|c|c|c|}
\hline Interval & $a$ & $\delta$ & $b$ & $A=e^{-a}$ & $\begin{aligned} E= & L A \\
& \times 10^{9}\end{aligned}$ & Replaced \\
\hline $\begin{array}{l}1 \\
2 \\
3 \\
4 \\
5 \\
6\end{array}$ & $\begin{array}{l}0.0 \\
0.5 \\
1.0 \\
1.5 \\
2.0 \\
2.5\end{array}$ & $\begin{array}{l}0.25 \\
0.25 \\
0.25 \\
0.25 \\
0.25 \\
0.25\end{array}$ & $\begin{array}{l}0.5 \\
1.0 \\
1.5 \\
2.0 \\
2.5 \\
3.0\end{array}$ & $\begin{array}{ll}1.000 & 000 \\
0.606 & 531 \\
0.367 & 879 \\
0.223 & 130 \\
0.135 & 335 \\
0.082 & 085\end{array}$ & $\begin{array}{r}2135 \\
1295 \\
785 \\
476 \\
289 \\
175\end{array}$ & $\begin{array}{l}X \times X \\
x \times x \\
x \times x \\
x \times x \\
x \times x\end{array}$ \\
\hline $\begin{array}{l}1.1 \\
1.2 \\
2.1 \\
2.2 \\
3.1 \\
3.2 \\
4.1 \\
4.2 \\
5.1 \\
5.2\end{array}$ & $\begin{array}{l}0.0 \\
0.2 \\
0.5 \\
0.7 \\
1.0 \\
1.2 \\
1.5 \\
1.7 \\
2.0 \\
2.2\end{array}$ & $\begin{array}{l}0.10 \\
0.15 \\
0.10 \\
0.15 \\
0.10 \\
0.15 \\
0.10 \\
0.15 \\
0.10 \\
0.15\end{array}$ & $\begin{array}{l}0.2 \\
0.5 \\
0.7 \\
1.0 \\
1.2 \\
1.5 \\
1.7 \\
2.0 \\
2.2 \\
2.5\end{array}$ & $\begin{array}{ll}1.000 & 000 \\
0.818 & 731 \\
0.606 & 531 \\
0.496 & 585 \\
0.367 & 879 \\
0.301 & 194 \\
0.223 & 130 \\
0.182 & 684 \\
0.135 & 335 \\
0.110 & 803\end{array}$ & $\begin{array}{r}10 \\
90 \\
6 \\
54 \\
4 \\
33 \\
2 \\
20 \\
1 \\
12\end{array}$ & \\
\hline
\end{tabular}

interpolation beyond the third decimal place in the argument, $n$ should be a factor of 1500 . For $n=10, \delta=0.15$; for $n=12, \delta=0.125$; and for $n=15, \delta=0.1$. Smaller values of $\delta$ can be used, but these are sufficient for present purposes. The numerical values for $T=5 \times 10^{-7}$ are shown in Table 1 for these three values of $n$. For 10 subintervals, the quadrature value is 0.950215596 and the adjusted value is 0.950212903 . The maximum error is $401 \times 10^{-9}$, and the discrepancy from the direct value is $29 \times 10^{-9}$. The discrepancy in the Simpson quadrature value, neglecting forcing errors of rounding, is $2664 \times 10^{-9}$. For 15 subintervals, the quadrature value is 0.950213462 , too large by $530 \times 10^{-9}$. The adjusted value is too small by 2 in the ninth decimal, whereas the possible error is 53. Each of the present method values has an error not exceeding the tolerance selected as $5 \times 10^{-7}$. Forcing errors of rounding have been neglected throughout.

If the intervals are not all taken as of the same length, more calculations are needed. In many applications, the gain in using intervals of the same length is less pronounced, especially if the limits of integration do not lend themselves to simple uniform subdivision. In the case of unequal intervals, the values of $L$ and $M$ vary and must be determined for each interval. As the total error can be determined only by adding the individual errors, a smaller tolerance must be selected for the subintervals. In each subinterval, $L \leqq T / A$, where $T$ is the subinterval tolerance. Then values of $L$ are determined for various values of $\delta$ until a value of $\delta$ can be selected to make the $L$ sufficiently small.

Frequently, especially when the values of the functions needed can be obtained simply, the method can be on a successive approximation basis. In this example, the interval of integration is first divided into six equal subintervals, and the maximum error is calculated for each, after which the errors guide further subdivision. Table 2 


\section{TABLE 3}

Simpson quadrature values for $I=\int_{0}^{3} e^{-x} d x$ and $T=5 \times 10^{-7}$

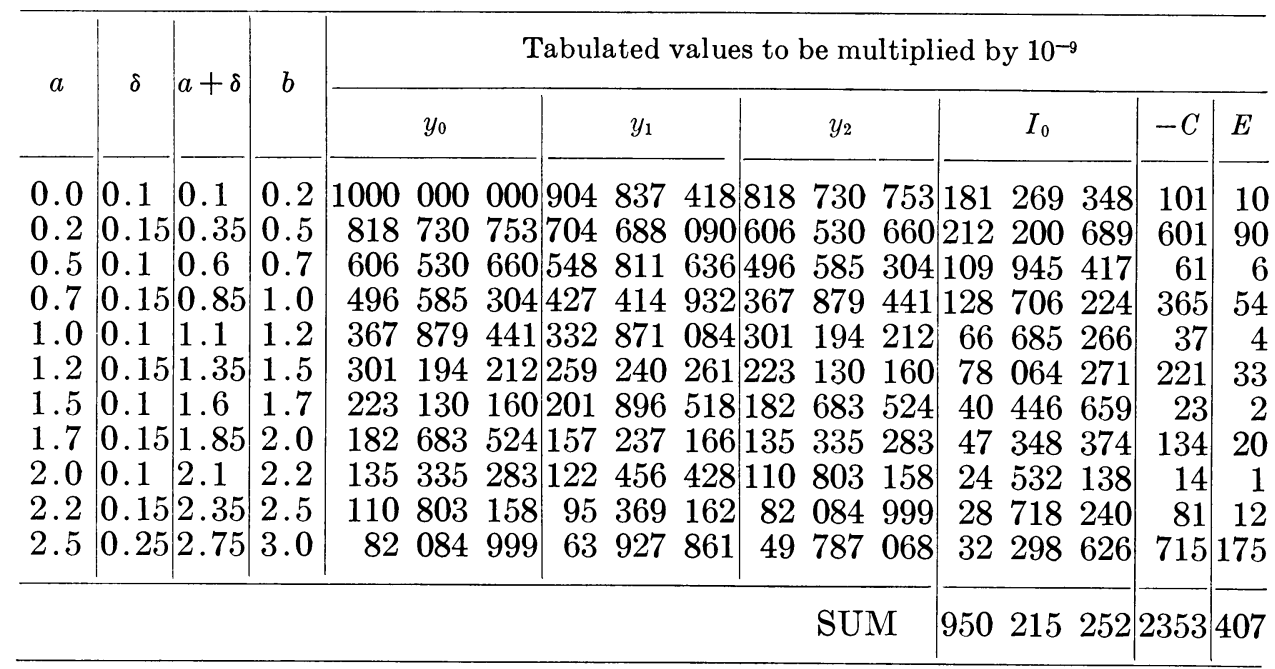

shows the first subdivision and the maximum error for each subinterval. If the total error tolerance is $5 \times 10^{-7}$, intervals 1,2 , and 3 must be subdivided, as the error term of each exceeds the tolerance. Table 2 indicates that interval 1 is replaced by intervals 1.1 and 1.2, interval 2 is replaced by intervals 2.1 and 2.2 , and interval 3 is replaced by intervals 3.1 and 3.2. At this stage of the calculation, intervals 1,2 , and 3 have been replaced and do not enter the calculations again. The sum of the error terms for all remaining intervals is $1137 \times 10^{-9}$, which exceeds the tolerance. Hence further subdivision is needed. The largest error term now occurs for interval 4 , which is therefore replaced by intervals 4.1 and 4.2. The error sum is now $683 \times$ $10^{-9}$, and another subdivision is needed. This time, interval 5 has the largest error term and is replaced by intervals 5.1 and 5.2. The sum of the error terms is now $403 \times 10^{-9}$, which is less than the tolerance. The method is progressive, and the accuracy can be improved until the forcing errors of rounding are of the same order as the individual error terms. The values of $L$ and $\delta^{5}$ could have been included in Table 2 , but it is preferable to compute them separately. For $\Delta=0.25, L=$ $2.134706 \times 10^{-6}$ and $\delta^{5}=9.765625 \times 10^{-4}$; for $\delta=0.15, L=1.093424 \times$ $10^{-7}$ and $\delta^{5}=7.593750 \times 10^{-5} ;$ and for $\delta=0.1, L=1.007050 \times 10^{-8}$ and $\delta^{5}=1 \times$ $10^{-5}$.

After the subdivision has been determined, the quadrature is performed in the usual manner, as shown in Table 3. For $\delta=0.25, M=8.715988 \times 10^{-6}$; for $\delta=$ $0.15, M=7.344076 \times 10^{-7}$; and for $\delta=0.1, M=1.010406 \times 10^{-7}$. The Simpson quadrature value is seen to be 0.950215252 , with a correction between 1946 and $2760 \times 10^{-9}$. The present method determines the integral value as 0.950212899 , with an error not over $407 \times 10^{-9}$. The discrepancies from the direct value are 2320 and 33, respectively, in the ninth decimal place, except for rounding errors.

Example 2. In the preceding example, the integrand was monotonic over the entire interval of integration, so that no internal extrema occurred on any subin- 
TABLE 4
Simpson quadrature intervals for $I=\int_{0}^{2} \sin x d x$ and $T=5 \times 10^{-7}$ $[n]=\times 10^{n}$

For $\delta=0.2, \quad \delta^{5} / 180=1.777778[-6]$

For $\delta=0.1, \quad \delta^{5} / 180=5.555556[-8]$

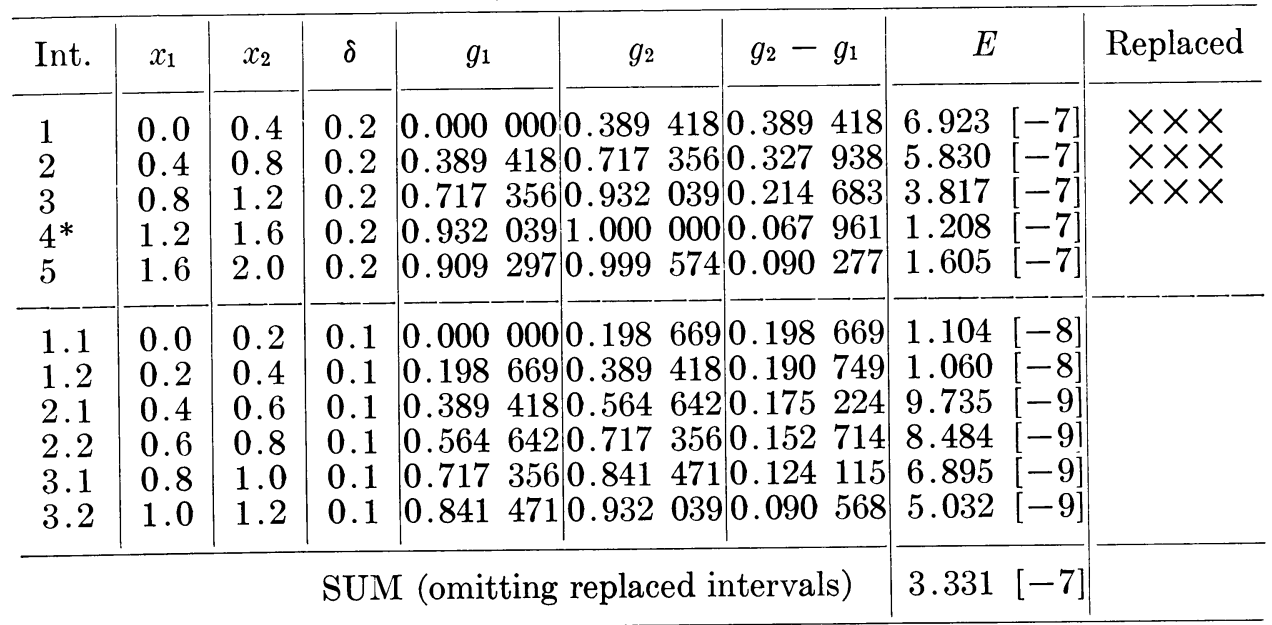

* This interval includes $x=\pi / 2=1.57$.

terval. In evaluating $I=\int_{0}^{2} \sin x d x$, the fourth derivative of the integrand is $g(x)=\sin x$, which has a maximum of 1 at $x=\Pi / 2=1.570796$. For $x \leqq 1.57$, $g(x)$ increases, so that on an interval $0 \leqq a \leqq x \leqq b \leqq 1.57, g_{1} \leqq g(a)=\sin a$ and $g_{2} \geqq \sin b$. On the contrary, for $1.57 \leqq a \leqq x \leqq b \leqq 3, g_{1} \leqq \sin b$ and $g_{2} \geqq \sin a$. For an interval containing $x=1.57, g_{2} \geqq 1$ and $g_{1}$ is less than or equal to the smaller of the two quantities $\sin a$ and $\sin b$.

For a preliminary estimate of $\delta$, it may be noted that $g_{1}=0$ and $g_{2}=1$ are suitable selections, so that for an error tolerance of $T, E=\delta^{5} / 180 \leqq T$. For $T=$ $5 \times 10^{-7}, \delta^{5} \leqq 1.8 \times 10^{-5}$ and $\delta \leqq 0.18$. For the choice $\delta=0.2$, the value of $\delta^{5} / 180$ is $(16 / 9) \times 10^{-6}$ and the determination of $E$ is shown in Table 4, with subdivisions as indicated. Directly, $I=1.416146837$. In Table 4 , the subscript 1 indicates a lower bound and the subscript 2 an upper bound. Table 4 indicates that the intervals 1 and 2 are too large, as $E>T$. Replacement of interval 1 by intervals 1.1 and 1.2 and interval 2 by intervals 2.1 and 2.2 reduces the total error in the seventh decimal place by $12.753-0.399=12.354$, so that at this point the total error cannot exceed the sum of the values of $E$ for the intervals $1.1,1.2,2.1,2.2,3,4$, and 5 , which is $7.029 \times 10^{-7}$, and hence exceeds $T$. Inspection of the table shows that the largest value of $E$ is in interval 3, which is therefore replaced by intervals 3.1 and 3.2 . The error now cannot exceed 3.33 in the seventh decimal place, and the subdivision is acceptable. For this subdivision, the quadrature is completed in Table 5, which shows that the Simpson value is 1.416154144 and the adjusted value is 1.416146962 , with an error not exceeding 333 in the ninth decimal place. The 


\section{TABLE 5}

Simpson quadrature values for $I=\int_{0}^{2} \sin x d x$ and $T=5 \times 10^{-7}$

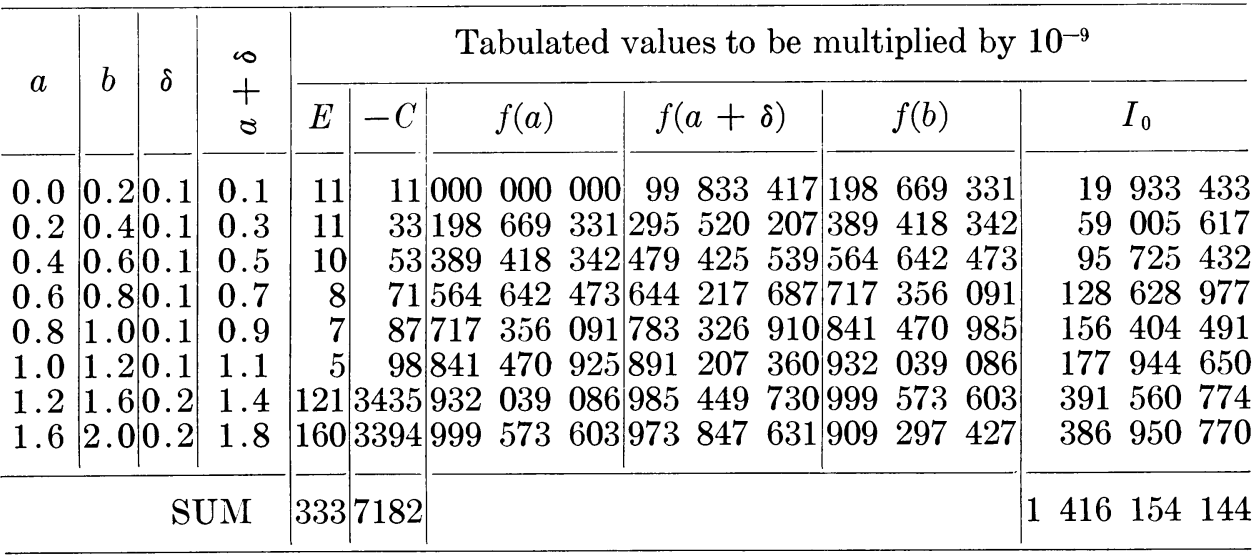

TABLE 6

Variations of components in $\int_{0}^{2} \sin x^{2} d x$

\begin{tabular}{c|c|c|c|c}
\hline$x$ & 0 & 1.253314 & 1.772454 & 2 \\
$z$ & 0 & $\Pi / 2$ & $\Pi$ & 4 \\
\hline $\sin z$ & 0 & +1 & 0 & -0.756803 \\
$\cos z$ & +1 & 0 & -1 & -0.653644 \\
\hline $16 z^{2}-12$ & -12 & & & +244 \\
$48 z$ & 0 & & & +192
\end{tabular}

Simpson value exceeds the direct value by 7307 in the ninth place, whereas the adjusted value exceeds it by 125 in that place, which is well within the tolerance.

Example 3. In the preceding examples, $g(x)=f(x)$, which usually is not true, and in each the integral could be evaluated directly, which often is not true. For the integral $I=\int_{0}^{2} \sin x^{2} d x, f(x)=\sin z$ and $g(x)=\left(16 z^{2}-12\right) \sin z-48 \cos z$, where $z$ has been written for $x^{2}$. The indefinite integral could not be found readily and is not used. On the range $0 \leqq x \leqq 2$, it is evident that $0 \leqq z \leqq 4$, and it is also true that $-0.756803 \leqq \sin z \leqq 1$ and $-1 \leqq \cos z \leqq 1$. The function $\sin z$ is 0 at $x=0$, is -0.756803 at $x=2$, and has a maximum value of 1 at $z=1.570796$, for which $x=1.253314$. The function $\cos z$ has a maximum of 1 at $x=0$, and a minimum of -1 at $z=3.141593$, for which $x=1.772454$. The factors $16 z^{2}-12$ and $48 z$ increase monotonically from $x=0$ to $x=2$. The extrema must be kept in mind for all intervals containing one or more of the values $0,1.253314,1.772454$, and 2 for $x$. Table 6 summarizes the variation of the various quantities, with each varying monotonically between the values shown. For the entire interval of integration, $-184.660 \leqq\left(16 z^{2}-12\right) \sin z \leqq+244,-192 \leqq 48 z \cos z \leqq 192$, 
TABLE 7

Quadrature intervals for $I=\int^{2} \sin x^{2} d x$

$\begin{aligned} \text { For } \delta & =0.2, \delta^{5} / 180=1.77778 \times 10^{-6} \\ \delta & =0.1, \delta^{5} / 180=5.555556 \times 10^{-8}\end{aligned}=\left(\delta^{5} / 180\right)\left(g_{2}-g_{1}\right)$

Notes: (1) This interval contains $z=1.5708$.

(2) This interval contains $z=3.1416$.

\begin{tabular}{|c|c|c|c|c|c|}
\hline Interval & 1 & 2 & 3 & 4 & 5 \\
\hline$x$ & $\begin{array}{l}0 \\
0.4\end{array}$ & $\begin{array}{l}0.4 \\
0.8\end{array}$ & $\begin{array}{l}0.8 \\
1.2\end{array}$ & $\begin{array}{l}1.2 \\
1.6\end{array}$ & $\begin{array}{l}1.6 \\
2.0\end{array}$ \\
\hline$z$ & $\begin{array}{l}0 \\
0.16\end{array}$ & $\begin{array}{l}0.16 \\
0.64\end{array}$ & $\begin{array}{l}0.64 \\
1.44\end{array}$ & $\begin{array}{l}1.44 \\
2.56\end{array}$ & $\begin{array}{l}2.56 \\
4.00\end{array}$ \\
\hline Note & & & & (1) & $(2)$ \\
\hline $\sin z$ & $\begin{array}{l}0 \\
0.15932\end{array}$ & $\begin{array}{l}0.15932 \\
0.59720\end{array}$ & $\begin{array}{l}0.59720 \\
0.99146\end{array}$ & $\begin{array}{l}0.99146 \\
1.00000\end{array}$ & $\begin{array}{r}-0.75680 \\
0.54936\end{array}$ \\
\hline $\cos z$ & $\begin{array}{l}0.98723 \\
1.00000\end{array}$ & $\begin{array}{l}0.80210 \\
0.98723\end{array}$ & $\begin{array}{l}0.13042 \\
0.80210\end{array}$ & $\begin{array}{r}-0.83559 \\
0.13042\end{array}$ & $\begin{array}{l}-1.00000 \\
-0.65364\end{array}$ \\
\hline $16 z^{2}-12$ & $\begin{array}{l}-12.000 \\
-11.590\end{array}$ & $\begin{array}{l}-11.590 \\
-5.4464\end{array}$ & $\begin{array}{c}-5.4464 \\
21.178\end{array}$ & $\begin{array}{l}21.178 \\
92.858\end{array}$ & $\begin{array}{l}92.858 \\
244\end{array}$ \\
\hline$\left(16 z^{2}-12\right) \sin z$ & $\begin{array}{l}-1.9118 \\
0\end{array}$ & $\begin{array}{l}-6.9215 \\
-0.86772\end{array}$ & $\begin{array}{c}-5.3999 \\
20.997\end{array}$ & $\begin{array}{l}20.997 \\
92.858\end{array}$ & $\begin{array}{r}-184.66 \\
134.04\end{array}$ \\
\hline$-48 z \cos z$ & $\begin{array}{l}-7.6800 \\
0\end{array}$ & $\begin{array}{l}-30.328 \\
-6.1601\end{array}$ & $\begin{array}{l}-55.441 \\
-4.0065\end{array}$ & $\begin{array}{c}-16.026 \\
102.68\end{array}$ & $\begin{array}{l}80.319 \\
192.00\end{array}$ \\
\hline$g$ & $\begin{array}{l}-9.5918 \\
0\end{array}$ & $\begin{array}{l}-37.250 \\
-7.0278\end{array}$ & $\begin{array}{r}-60.841 \\
16.990\end{array}$ & $\begin{array}{l}4.9710 \\
195.54\end{array}$ & $\begin{array}{r}-104.34 \\
326.04\end{array}$ \\
\hline$g_{2}-g_{1}$ & 9.5918 & 30.222 & 77.831 & 190.57 & 430.38 \\
\hline$\delta$ & 0.2 & 0.2 & 0.2 & 0.2 & 0.2 \\
\hline$E \times 10^{8}$ & 1705 & 5373 & 13837 & 33879 & 76512 \\
\hline
\end{tabular}

$g_{1} \leqq-376.660$, and $g_{2} \geqq+436$. Hence $g_{2}-g_{1} \geqq 812660$ and $\delta=1$, so that $E=$ 4.515 and $I_{0}=+0.8697$. Accordingly, the possible error is over five times the Simpson value, and subdivision is needed. For five equal intervals, the error terms are shown in Table 7, in which the intervals follow from left to right. The total error does not exceed 0.00131306 . If intervals 4 and 5 are subdivided into two equal subintervals each, omitted calculations show that the total error will not exceed 0.00023980 . Then replacing interval 3 by two equal subintervals reduces the possible error to 0.00010580 . The process continues until the sum of the error terms is acceptably small or the forcing errors of rounding are significant, after which the quadrature values and the correction terms are computed as for the previous ex- 
amples. Table 7 shows only the first five subintervals, as the calculations follow without introduction of new considerations. It should be noted that in Table 7, the minima of the functions in a selected column do not always come from the same value of $x$, but each is selected by inspection from the possible values. Also, intervals 4 and 5 have internal extrema that override the values at the ends of the interval. This also occurs for some of the replacing subintervals.

Example 4. In the preceding examples, the integrand has a single factor. If $f=u v$, where $u$ and $v$ are functions of $x$, the value of $f^{i v}(x)$ is $g(x)=u v^{i v}+4 u^{\prime} v^{\prime \prime \prime}+$ $6 u^{\prime \prime} v^{\prime \prime}+4 u^{\prime \prime \prime} v^{\prime}+u^{i v} v$, and bounds of each term can be selected from the product of the bounds of its factors. No numerical example is included as no new procedures are in volved.

4. Summary. The method of quadrature discussed in this paper has several important aspects:

a. The error terms are used to select subintervals to assure a negligible overall error.

b. Bounds are used instead of extrema when the latter are not readily available.

c. The lower and upper bounds of the error terms are used, thus determining a mean value of the error and an adjusted quadrature value.

d. The bounds of a product are selected from the products of the bounds of its factors.

e. After the subintervals have been selected, the quadrature formula is applied and the value adjusted by addition of the total of the corrections for the separate subintervals.

f. The method is not restricted to the use of Simpson's formula which was selected to illustrate the method. However, details will vary with the formula.

U. S. Geological Survey

Washington, D. C.

1. J. F. Steffensen, Interpolation, Chelsea Publishing Co., New York, 1927.

2. James B. Scarborough, Numerical Mathematical Analysis, 2nd Edition, Johns Hopkins Press, Baltimore, 1950. 